\title{
PROPRIEDADES FÍSICAS DE LIPÍDIOS ESTRUTURADOS OBTIDOS DE MISTURAS DE GORDURA DO LEITE E ÓlEO DE MILHO ${ }^{1}$
}

\author{
Juliana Neves RODRIGUES ${ }^{2}$, Luiz Antonio GIOIELLI ${ }^{2, *}$ e Carolina ANTON ${ }^{2}$
}

\begin{abstract}
RESUMO
Através da mistura e interesterificação de óleos e gorduras podem ser obtidos lipídios estruturados, que são compostos que apresentam características fisicas, quimicas e nutricionais diferentes das dos lipídios que lhes deram origem. Esses novos compostos podem apresentar capacidade de reduzir o risco de doenças, sendo então chamados de "alimentos funcionais". O objetivo deste estudo foi avaliar as propriedades físicas de lipídios estruturados obtidos por interesterificação química a partir de misturas de gordura do leite e óleo de milho. Foram preparadas quatro misturas, nas proporções de 80:20, 60:40, 40:60 e 20:80 de gordura do leite e óleo de milho, respectivamente. As amostras foram submetidas à interesterificação química com catalisador metóxido de sódio. As misturas e os lipídios estruturados obtidos foram avaliados quanto à consistência, conteúdo de gordura sólida e composição em ácidos graxos. Foram obtidos lipídios estruturados contendo teores de até $22 \%$ de ácido linoléico pela adição de até $40 \%$ de óleo de milho à gordura do leite, que originalmente possuía apenas cerca de $2 \%$ deste ácido graxo. A consistência e o conteúdo de gordura sólida foram dependentes da gordura do leite e da interação desta com o óleo de milho. Os coeficientes relativos às interações entre os componentes das misturas foram negativos, demonstrando efeito antagônico, característico de interações eutéticas entre gorduras. Os resultados mostraram relação linear significativa entre o conteúdo de gordura sólida e a consistência das amostras.

Palavras-chave: gordura do leite; óleo de milho; mistura; interesterificação; propriedades físicas.
\end{abstract}

\section{SUMMARY}

PHYSICAL PROPERTIES OF STRUCTURED LIPIDS OBTAINED FROM MILKFAT AND CORN OIL BLENDINGS. Structured lipids can be obtained by blending and chemical interesterification of fats and oils, resulting in products with physical, chemical and nutritional properties that are different from the original lipids. These new products can be able to reduce risk of diseases, being called functional foods. The objective of this study was to evaluate the physical properties of structured lipids obtained through chemical interesterification of blendings of milkfat and corn oil. Four blendings were prepared in the proportions 80:20, 60:40, 40:60 e 20:80 of milkfat and corn oil, respectively. The samples were submitted to chemical interesterification with sodium methoxide as catalyst. The blendings and the structured lipids were evaluated as for consistency, solid fat content and fatty acid composition. Structured lipids with contents of up to $22 \%$ of linoleic acid were obtained by the addition of up to $40 \%$ of corn oil to milkfat, which originally had just about $2 \%$ of this fatty acid. Consistency and solid fat content were dependent on milkfat and on its interaction with corn oil. The significant coefficients related to interactions between the fats were always negative, demonstrating an antagonic effect, characteristic of eutectic interactions between fats. A significant linear relationship between solid fat content and consistency was obtained.

Keywords: milkfat; corn oil; blending; interesterification; physics properties.

\section{1 - INTRODUÇÃo}

Alimentos funcionais são semelhantes em aparência ao alimento convencional, consumidos como parte da dieta usual, capazes de produzir demonstrados efeitos metabólicos ou fisiológicos úteis na manutenção de boa saúde física e mental, podendo auxiliar na redução do risco de doenças crônico-degenerativas, além das suas funções nutricionais básicas. Mais importante, entretanto, é o potencial dos alimentos funcionais diminuirem as doenças, promoverem a saúde e reduzirem os custos com os cuidados com a saúde. Obviamente, todos os alimentos são funcionais, visto que proporcionam sabor, aroma ou valor nutritivo. Alimento funcional, porém, não deve ser confundido com medicamento. O primeiro está principalmente ligado à nutrição, tem como função manter a saúde e é recomendado à população em geral, pois não deve apresentar riscos, enquanto que o segundo está ligado à área médica, tem como objetivo curar doenças e é receitado especificamente ao individuo doente, pois apresenta uma

1. Recebido para publicação em 19/12/2001. Aceito para publicação em 18/09/2002 (000788).

2. Departamento de Tecnologia Bioquímico-Farmacêutica, Faculdade de Ciências Farmacêuticas, USP. Caixa Postal 66083, CEP 05315-970, São Paulo-SP,Brasil. E-mail: lagio@usp.br

* A quem a correspondencia deve ser enviada. relação risco/beneficio [18, 24].

O consumidor está se tornando mais consciente da relação entre alimentação e doenças. Assim, vem crescendo a demanda por informação sobre os efeitos dos nutracêuticos e alimentos funcionais sobre a saúde [11]. Os alimentos funcionais normalmente custam mais que as versões tradicionais. Assim, para ter grande aceitação, devem oferecer beneficios à saúde que sejam claros para o consumidor [10, 19].

A gordura é um importante componente da dieta, entretanto, a dieta ocidental moderna não pode ser qualificada como balanceada, pois contém muita gordura e apresenta desequilibrio no tipo de gordura consumida [12]. Uma alimentação rica em gorduras saturadas pode provocar doenças cardiovasculares, que são a principal causa de morte no Brasil, representando cerca de 40\% das mortes de pessoas com mais de 45 anos, de acordo com a estatística anual da Organização Mundial de Saúde [26].

A gordura do leite, como todos os óleos e gorduras provenientes de fontes naturais, é uma mistura de vários tipos de triacilgliceróis (mais de 100.000 diferentes tipos com faixa de ponto de fusão variando de -40 a $40^{\circ} \mathrm{C}$ ), que contêm cerca de 400 diferentes ácidos graxos [31]. Essa sua composição tão variada é responsável pelo sabor e propriedades físicas únicas. Por muitos anos, contudo, 
várias discussões foram levantadas quanto ao valor da gordura do leite em relação à saúde. Ela tem sido vista como prejudicial por conter quantidades razoáveis de colesterol e ácidos graxos saturados, principalmente os ácidos láurico, mirístico e palmítico. Assim, nas duas últimas décadas o consumo mundial de manteiga tem diminuído [2, 3]. Sob temperatura de refrigeração doméstica $\left(\sim 10^{\circ} \mathrm{C}\right)$, a manteiga essencialmente se comporta como um sólido e tem pouca espalhabilidade, enquanto que à temperatura ambiente $\left(21-25^{\circ} \mathrm{C}\right)$, ocorre separação de óleo e exsudação. Sua espalhabilidade ideal ocorre por volta de $15^{\circ} \mathrm{C}$, quando seu conteúdo de gordura sólida está em torno de $30 \%$ [31]. A espalhabilidade é significativamente influenciada pela firmeza da manteiga, que é determinada, em parte, pelo seu conteúdo de gordura sólida e pela composição em ácidos graxos. Quanto maiores forem o conteúdo de gordura sólida e o teor de ácidos graxos saturados da gordura do leite, mais firme será a manteiga, reduzindo sua espalhabilidade [23].

O crescimento da demanda do consumidor por "spreads" (margarinas ou cremes vegetais) que, além de serem agradáveis ao paladar, podem ser usados no preparo de pratos e possuem boas propriedades físicas, como textura e cremosidade, tem servido como um impulso para a produção industrial de manteigas modificadas e produtos à base de manteiga. Muitos produtos estão presentes nos supermercados de todo o mundo e novos produtos estão surgindo constantemente [31].

A mistura de manteiga com óleos vegetais, como os de milho, canola, girassol, soja, entre outros, que são liquidos à temperatura de refrigeração pode levar a produtos que equilibram a nutrição com as características organoléticas desejáveis e custos de produção mais baixos [31]. A principal tendência está em incorporar o máximo possivel de óleo liquido monoinsaturado ou poliinsaturado a esses produtos por razões nutricionais. Contudo, isso leva a dificuldades técnicas quanto à estabilidade de suas características de espalhabilidade e suas propriedades de textura [27].

As tecnologias para modificação de óleos e gorduras que têm obtido sucesso comercial fazem uso de mistura, hidrogenação, fracionamento e interesterificação, química ou enzimática [2]. O uso da interesterificação é uma tendência que se apresenta como uma opção na indústria de gorduras para alterar propriedades de espalhabilidade, pois pode modificar substancialmente as propriedades físicas das gorduras [28]. A interesterificação envolve a troca e a redistribuição dos ácidos graxos entre os triacilgliceróis. O produto resultante apresenta a mesma composição em ácidos graxos totais do material inicial, mas a composição em triacilgliceróis e as propriedades físicas são alteradas [13, 20, 33, 35]. A interesterificação química leva a uma distribuição ao acaso dos ácidos graxos na cadeia do triacilglicerol. A interesterificação enzimática também é especifica, pois utilizam-se lipases seletivas [22]. Enquanto a interesterificação enzimática é um processo que está reservado ao futuro, no presente a interesterificação química consiste em um processo de custo mais baixo para utilização em escala industrial. A substituição de ácidos graxos saturados na posição sn-2 do triacilglicerol da gordura do leite por ácidos graxos hipocolesterolêmicos, derivados de óleos vegetais, é uma conseqüência nutricional importante da interesterificação [31].

O óleo de milho é considerado de primeira qualidade pelo consumidor, pois tem sabor agradável, levemente adocicado. Devido a sua estabilidade inerente, tem longa vidade-prateleira e é resistente a transformações sob condições adversas, como as encontradas durante a fritura. O óleo de milho pode ter um papel muito importante na dieta humana, pois é facilmente digerido, oferece ácidos graxos essenciais e vitamina $\mathrm{E}$ e representa uma fonte rica de ácidos graxos poliinsaturados, o que ajuda a regular os niveis de colesterol no sangue e a pressão arterial. Uma colher de sopa ( 14 g ou 120kcal) de óleo de milho satisfaz a necessidade diária de ácidos graxos essenciais para uma criança ou um adulto saudáveis [20].

O objetivo deste estudo foi avaliar as propriedades fisicas de lipídios estruturados obtidos por interesterificação química a partir de misturas de gordura do leite e óleo de milho.

\section{2 - MATERIAL E MÉTODOS}

\section{1 - Material}

A gordura do leite foi obtida a partir de manteiga comercial tipo extra, por fusão completa à temperatura de $60-70^{\circ} \mathrm{C}$ em forno de microondas e filtração em papel filtro qualitativo de porosidade $3 \mu \mathrm{m}$, realizada em estufa a $50^{\circ} \mathrm{C}$. O óleo de milho refinado comercial foi utilizado sem tratamento adicional.

\section{2 - Preparação das misturas}

Para o estudo das interações em misturas binárias de gorduras realizou-se um planejamento de 6 experimentos (Tabela 1). A gordura do leite e o óleo de milho são representados por $\mathrm{x} 1 \mathrm{e} \mathrm{x} 2$, respectivamente, sendo que $\mathrm{x} 1+\mathrm{x} 2=1$ ou $100 \%$. Duas amostras representam os componentes isoladamente e quatro são formadas por misturas binárias. As amostras foram preparadas por mistura nas proporções mencionadas, após fusão completa à temperatura de $60-70^{\circ} \mathrm{C}$ e novamente solidificadas, sendo armazenadas sob resfriamento.

TABELA 1. Planejamento experimental das misturas.

\begin{tabular}{ccc} 
& \multicolumn{2}{c}{ Componentes (proporção $\mathrm{p} / \mathrm{p}$ ) } \\
Amostra (n.) & $\mathrm{x}_{1}$ & $\mathrm{x}_{2}$ \\
1 & 1 & 0 \\
2 & 0,8 & 0,2 \\
3 & 0,6 & 0,4 \\
4 & 0,4 & 0,6 \\
5 & 0,2 & 0,8 \\
6 & 0 & 1 \\
\hline
\end{tabular}




\section{3 - Interesterificação química}

Foram interesterificados $350 \mathrm{~g}$ de cada amostra, previamente seca em balão de fundo redondo, sob pressão reduzida, acoplado a um rotoevaporador, em banho de água a $95^{\circ} \mathrm{C}$. A essa porção foi misturado $0,5 \%(\mathrm{~m} / \mathrm{m}) \mathrm{de}$ catalisador metóxido de sódio. Este foi obtido por evaporação sob pressão reduzida do álcool da solução de metóxido de sódio a $30 \%$, em banho de água a $80^{\circ} \mathrm{C}$. A reação de interesterificação foi realizada sob agitação constante, sob pressão reduzida, em balão de três bocas, por 1 hora a $65-70^{\circ} \mathrm{C}$. Para interromper a reação adicionou-se água destilada $(5 \mathrm{~mL})$. Para minimizar o escurecimento decorrente da reação e para reter a umidade foram adicionados sílica em pó e sulfato de sódio anidro, respectivamente. Os reagentes foram filtrados a quente utilizando-se papel filtro.

\section{4 - Composição em ácidos graxos}

A análise de ácidos graxos foi realizada por cromatografia em fase gasosa, segundo normas da AOCS, [1], método Ce 1-62. Foi empregado cromatógrafo a gás Varian GC, modelo 3400CX, equipado com detector de ionização de chama e "Workstation Star Chromatography". Foi utilizada coluna capilar de silica fundida CP WAX 52CB (Chrompack), com 30 metros de comprimento x 0,25mm de diâmetro interno e contendo $0,25 \mu \mathrm{m}$ de polietilenoglicol. As condições foram: injeção split, razão de 50:1; temperatura da coluna: $75^{\circ} \mathrm{C}$ por $3 \mathrm{~min}$, programada até $150^{\circ} \mathrm{C}$ numa razão de $37,5^{\circ} \mathrm{C} / \mathrm{min}$ e novamente programada até $215^{\circ} \mathrm{C}$ numa razão de $3^{\circ} \mathrm{C} / \mathrm{min}$; gás de arraste: hélio, numa vazão de $1,5 \mathrm{~mL} / \mathrm{min}$; gás make-up: hélio, a $30 \mathrm{~mL} / \mathrm{min}$; temperatura do injetor: $250^{\circ} \mathrm{C}$; temperatura do detector: $280^{\circ} \mathrm{C}$. A composição qualitativa foi determinada por comparação dos tempos de retenção dos picos com os dos respectivos padrões de ácidos graxos. A composição quantitativa foi realizada por normalização de área, sendo expressa como porcentagem em massa. Os ésteres metilicos de ácidos graxos para a análise foram obtidos de acordo com o método descrito por HARTMAN \& LAGO [17].

\section{5 - Consistência}

Foi determinada utilizando o equipamento analisador de textura TA-XT2, fabricado pela SMS (STABLE MICRO SYSTEMS), controlado por microcomputador. As amostras foram aquecidas em forno de microondas, até a temperatura de $60-70^{\circ} \mathrm{C}$, para a fusão completa dos cristais, e acondicionadas em béqueres de $250 \mathrm{~mL}$. O condicionamento foi efetuado por 24 horas em geladeira comum $\left(5-8^{\circ} \mathrm{C}\right)$ para a recristalização da gordura e posteriormente por 24 horas em estufa a temperatura controlada. Foi utilizado cone de acrílico com ponta não truncada e ângulo de $45^{\circ}$. Os testes foram operados nas seguintes condições: retorno ao início; distância $=10 \mathrm{~mm}$; velocidade $=2 \mathrm{~mm} / \mathrm{s}$; tempo $=5 \mathrm{~s}$; determinação da força em compressão (gf); triplicata: 3 compressões em pontos diferentes para cada amostra [7].

As amostras foram analisadas quanto ao "yield value". Para o cálculo do "yield value" foi utilizada a seguinte equação, proposta por HAIGHTON [16]:

$$
\begin{aligned}
& C=K \frac{W}{p^{1,6}} \\
& \text { onde: } \\
& \mathrm{C}=\text { "yield value" }\left(\mathrm{gf} / \mathrm{cm}^{2}\right) \\
& \mathrm{K}=\text { fator dependente do ângulo do cone } \\
& \mathrm{W}=\text { força em compressão (gf), para tempo de } 5 \mathrm{~s} . \\
& \mathrm{p}=\text { profundidade de penetração }(0,1 \mathrm{~mm}) .
\end{aligned}
$$

\section{6 - Conteúdo de gordura sólida}

O conteúdo de gordura sólida, analisado por ressonância nuclear magnética de baixa resolução $(20 \mathrm{MHz})$, utilizando aparelho Maran Ultra Bench Top NMR, foi determinado segundo normas da AOCS [1], método Cd 16b-93, procedimento em série.

\section{7 - Análise estatística}

Aos resultados obtidos referentes as misturas e as reações de interesterificação foi aplicado um modelo de regressão múltipla do tipo quadrático, representado pela seguinte equação:

$$
y=\beta_{1} x_{1}+\beta_{2} x_{2}+\beta_{12} x_{1} x_{2}
$$

onde:

$$
y=\text { resposta }
$$

$\beta=$ coeficientes gerados por regressão múltipla;

$x=$ proporção do componente.

Utilizou-se o aplicativo Statgraphics versão 2.6, que gerou os coeficientes para o modelo, além de apresentar seus niveis de significância, coeficientes de determinação e análise de variância.

\section{3 - RESULTADOS E DISCUSSÃO}

Os resultados da composição em ácidos graxos das amostras estão apresentados na Tabela 2, onde são listados 44 diferentes ácidos graxos, incluindo alguns não identificados. Isto confirma uma das características da gordura do leite, que é a grande variedade de ácidos graxos, com o número de carbonos das cadeias variando bastante, desde ácidos graxos de cadeia curta até os de cadeia longa [21].

A composição em ácidos graxos da gordura do leite está de acordo com a literatura [15]. Os ácidos graxos saturados constituem a grande maioria. Predominam os ácidos graxos palmítico, oléico, mirístico e esteárico. Foi detectada a presença do ácido butírico, característico da gordura do leite e comumente utilizado para quantificar a presença de manteiga em alimentos [29]. Os resultados também estão de acordo com a Portaria n. 146/96 [4], que estabelece valores para as seguintes relações entre os principais ácidos graxos da gordura do leite: 14:0/18:1, 14:0/12:0, 12:0/10:0 e 10:0/8:0. 
TABELA 2. Composição em ácidos graxos das amostras e teores de ácidos graxos saturados e insaturados.

\begin{tabular}{|c|c|c|c|c|c|c|c|}
\hline $\begin{array}{c}\text { Ácido Graxo } \\
(\%)\end{array}$ & $\begin{array}{c}\text { Tempo de } \\
\text { retenção (min) }\end{array}$ & $\begin{array}{c}\text { Amostra } 1 \\
(100: 0)^{*}\end{array}$ & $\begin{array}{c}\text { Amostra } 2 \\
(80: 20)^{*}\end{array}$ & $\begin{array}{c}\text { Amostra } 3 \\
(60: 40)^{\star}\end{array}$ & $\begin{array}{c}\text { Amostra } 4 \\
(40: 60)^{*}\end{array}$ & $\begin{array}{c}\text { Amostra } 5 \\
(20: 80)^{*}\end{array}$ & $\begin{array}{c}\text { Amostra } 6 \\
(0: 100)^{*}\end{array}$ \\
\hline $4: 0$ & 1,5 & 0,8 & 0,8 & 0,6 & 0,3 & 0,2 & - \\
\hline $6: 0$ & 2,8 & 1,5 & 1,4 & 1,1 & 0,6 & 0,3 & - \\
\hline $8: 0$ & 4,7 & 1,1 & 1,0 & 0,8 & 0,4 & 0,2 & - \\
\hline $10: 0$ & 6,1 & 2,6 & 2,4 & 1,7 & 1,1 & 0,6 & - \\
\hline $10: 1$ & 6,5 & 0,3 & 0,3 & - & - & - & - \\
\hline $12: 0$ & 7,9 & 3,1 & 2,7 & 1,9 & 1,3 & 0,7 & - \\
\hline $12: 1$ & 8,5 & 0,1 & - & - & - & - & - \\
\hline $14: 0$ iso & 9,8 & 0,2 & - & - & - & - & - \\
\hline 14:0 & 10,6 & 10,6 & 8,9 & 6,4 & 4,3 & 2,2 & - \\
\hline $14: 1 \mathrm{n}-9$ & 11,2 & 1,0 & 0,8 & 0,6 & 0,4 & 0,2 & - \\
\hline n.i. & 11,5 & 0,4 & 0,3 & 0,1 & 0,1 & - & - \\
\hline n.i. & 11,7 & 0,6 & 0,5 & 0,4 & 0,2 & - & - \\
\hline $15: 0$ & 12,4 & 1,2 & 1,0 & 0,7 & 0,5 & 0,3 & - \\
\hline $16: 0$ iso & 13,4 & 0,3 & 0,2 & - & - & - & - \\
\hline 16:0 & 14,6 & 29,3 & 26,4 & 23,0 & 19,7 & 16,5 & 13,0 \\
\hline n.i. & 14,8 & 0,2 & 0,1 & - & - & - & - \\
\hline $16: 1 \mathrm{n}-9$ & 15,0 & 1,6 & 1,3 & 1,0 & 0,7 & 0,4 & - \\
\hline n.i. & 15,5 & 0,5 & 0,4 & 0,3 & 0,2 & - & - \\
\hline n.i. & 15,9 & 0,5 & 0,4 & 0,3 & 0,2 & - & - \\
\hline n.i. & 16,5 & 0,2 & - & - & - & - & - \\
\hline $17: 0$ & 16,6 & 0,7 & 0,6 & 0,4 & 0,3 & 0,2 & - \\
\hline $17: 1 n-9$ & 17,1 & 0,3 & 0,3 & - & 0,1 & - & - \\
\hline 18:0 & 19,2 & 11,8 & 9,7 & 8,0 & 6,0 & 4,0 & 2,1 \\
\hline $18: 1 n-9$ & 19,7 & 21,3 & 23,6 & 26,8 & 29,2 & 31,4 & 34,0 \\
\hline $18: 1 \mathrm{n}-9$ trans & 19,8 & 3,2 & 2,6 & 2,1 & 1,8 & 1,2 & 0,3 \\
\hline n.i. & 19,9 & 0,8 & 0,6 & 0,5 & - & - & - \\
\hline n.i. & 20,1 & 0,3 & 0,3 & - & - & - & - \\
\hline n.i. & 20,4 & 0,4 & - & - & - & - & - \\
\hline n.i. & 20,4 & 0,1 & - & - & - & - & - \\
\hline $18: 2 n-6$ & 20,6 & 1,9 & 11,5 & 21,6 & 30,9 & 40,0 & 48,7 \\
\hline n.i. & 21,1 & 0,4 & 0,3 & 0,1 & - & - & - \\
\hline $18: 3 n-6$ & 24,6 & 0,1 & - & - & - & - & - \\
\hline n.i. & 22,0 & 0,1 & - & - & - & - & - \\
\hline $18: 3 n-3$ & 22,2 & 0,6 & 0,6 & 0,7 & 0,8 & 0,8 & 0,8 \\
\hline $18: 4 n-3$ & 22,8 & 1,0 & 0,8 & 0,6 & 0,4 & 0,2 & - \\
\hline 20:0 & 24,0 & 0,2 & 0,2 & 0,3 & 0,4 & 0,4 & 0,5 \\
\hline n.i. & 24,3 & 0,2 & - & - & 0,1 & 0,2 & - \\
\hline $20: 1$ & 24,4 & - & - & - & - & - & 0,3 \\
\hline n.i. & 26,4 & 0,1 & - & - & - & - & - \\
\hline n.i. & 26,9 & 0,2 & - & - & - & - & - \\
\hline $22: 0$ & 29,2 & - & - & - & - & - & 0,1 \\
\hline n.i. & 31,9 & 0,1 & - & - & - & - & - \\
\hline n.i. & 32,9 & 0,1 & - & - & - & - & - \\
\hline $24: 0$ & 37,2 & - & - & - & - & - & 0,2 \\
\hline Total & & 100,0 & 100,0 & 100,0 & 100,0 & 100,0 & 100,0 \\
\hline Saturados & & 63,1 & 55,1 & 44,9 & 34,9 & 25,6 & 15,9 \\
\hline Monoinsaturados & & 28,1 & 29,1 & 30,5 & 32,2 & 33,2 & 34,6 \\
\hline Poliinsaturados & & 3,6 & 12,9 & 22,9 & 32,1 & 41,0 & 49,5 \\
\hline
\end{tabular}

*Os números entre parênteses referem-se a proporção entre as gorduras (gordura do leite:óleo de milho). 
A composição do óleo de milho está em conformidade com o CODEX ALIMENTARIUS (1979) e a legislação brasileira [5]. O ácido linoléico $(\omega-6)$, presente em quase $50 \%$ da composição do óleo, o caracteriza como uma gordura do grupo do ácido oléico/linoléico. Não foram encontrados ácidos graxos de cadeia curta e os saturados aparecem em pequena quantidade, características estas tipicas de óleos vegetais.

A adição de óleo de milho à gordura do leite provocou aumento no teor de ácidos graxos poliinsaturados $\omega-6$. Os lipídios estrutrados contendo $80 \%$ de gordura do leite e $20 \%$ de óleo de milho apresentaram $11,5 \%$ de ácido linoléico, enquanto os que contém $60 \%$ de gordura do leite e $40 \%$ de óleo de milho apresentaram $21,6 \%$ deste ácido graxo, contra apenas 1,9\% da gordura do leite pura. Desta maneira, o grau de saturação da gordura do leite em um spread pode ser diminuído pela adição de um óleo vegetal, o que contribuiria não só para melhoria nas características físicas do produto, como também nas propriedades nutricionais e para menores custos de produção [31].

A Tabela 3 apresenta a consistência das amostras antes e depois da interesterificação, calculada como "yield value", nas temperaturas de $\mathrm{O}$ a $30^{\circ} \mathrm{C}$. Os resultados mostraram que a adição de óleo de milho provocou diminuição na consistência de todas as amostras, pelo fato de ser um óleo líquido e assim provocar a diluição da rede cristalina da gordura do leite. A adição de apenas $20 \%$ de óleo de milho já provocou grande queda na consistência da gordura do leite, o que pode ser devido a uma rede cristalina estruturalmente mais frágil [32].

Também pôde-se observar que a consistência é diminuída com o aumento da temperatura, que provoca a fusão gradual dos cristais e consequentemente a destruição da rede cristalina, o que confere plasticidade à gordura [8]. Nenhuma das amostras apresentou consistência a $30^{\circ} \mathrm{C}$. Não foi possivel determinar a consistência da amostra 6 , pois o óleo de milho é liquido nas temperaturas em que foi analisado.

A Figura 1 representa a consistência das amostras 1,2 e 3 antes e depois da interesterificação, em função da temperatura. A interesterificação provocou aumento na consistência das amostras na maioria dos casos. Todavia, principalmente a temperaturas mais baixas $(0$ e $5^{\circ} \mathrm{C}$ ), houve diminuição na consistência, o que pode ser devido à incorporação pelos triacilgliceróis dos ácidos graxos insaturados do óleo de milho [32]. Esta diferença de comportamento da propriedade de consistência em função da temperatura pode ser explicada pela estrutura da rede cristalina das gorduras, que pode cristalizar em diferentes formas polimórficas, dependendo do tratamento térmico a que são submetidas, ou simplesmente da temperatura de cristalização [34].

A Tabela 4 apresenta os coeficientes obtidos por regressão múltipla para a propriedade de consistência das amostras, antes e depois da interesterificação. A consistência foi dependente da gordura do leite e da interação com o óleo de milho. Os coeficientes $\beta_{12}$ relativos à interação entre os componentes são negativos para todas as temperaturas analisadas, o que revela interação eutética entre a gordura do leite e o óleo de milho, antes e depois do rearranjo. A interação eutética ocorre em função do empacotamento das moléculas ser dificultado, por diferenças no tamanho molecular e de polimorfismo [30, 36]. A gordura do leite contém ácidos graxos de cadeia curta e média e cristaliza na forma beta-prima, ao passo que o óleo de milho contém ácidos graxos de cadeia longa e tende a cristalizar na forma beta [6, 37].

TABELA 3. Consistência das amostras $\left(\mathrm{gf} / \mathrm{cm}^{2}\right)$ antes e depois da interesterificação.

\begin{tabular}{|c|c|c|c|c|c|c|c|c|c|c|c|c|}
\hline \multirow[t]{2}{*}{$\begin{array}{c}\text { Temperatura } \\
\left({ }^{\circ} \mathrm{C}\right)\end{array}$} & \multicolumn{2}{|c|}{$\begin{array}{l}\text { Amostra } 1 \\
(100: 0)^{*}\end{array}$} & \multicolumn{2}{|c|}{$\begin{array}{c}\text { Amostra } 2 \\
(80: 20)^{\star}\end{array}$} & \multicolumn{2}{|c|}{$\begin{array}{c}\text { Amostra } 3 \\
(60: 40)^{\star}\end{array}$} & \multicolumn{2}{|c|}{$\begin{array}{c}\text { Amostra } 4 \\
(40: 60)^{*}\end{array}$} & \multicolumn{2}{|c|}{$\begin{array}{l}\text { Amostra } 5 \\
(20: 80)^{\star}\end{array}$} & \multicolumn{2}{|c|}{$\begin{array}{c}\text { Amostra } 6 \\
(0: 100)^{\star}\end{array}$} \\
\hline & Antes & Depois & Antes & Depois & Antes & Depois & Antes & Depois & Antes & Depois & Antes & Depois \\
\hline 0 & 20003 & 8891 & 7297 & 6247 & 2825 & 2855 & 742 & 557 & 154 & 203 & 0 & 0 \\
\hline 5 & 11610 & 8860 & 4832 & 5204 & 1622 & 1576 & 381 & 463 & 0 & 0 & - & - \\
\hline 10 & 6360 & 6490 & 3240 & 4241 & 1119 & 978 & 169 & 274 & - & 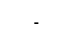 & - & 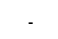 \\
\hline 15 & 3416 & 5050 & 1446 & 2033 & 293 & 387 & 0 & 0 & - & - & - & - \\
\hline 20 & 607 & 4176 & 259 & 1380 & 0 & 249 & - & - & - & - & - & - \\
\hline 25 & 260 & 1710 & 0 & 659 & - & 0 & - & - & - & - & - & - \\
\hline 30 & 0 & 0 & - & 0 & - & - & - & - & - & - & - & - \\
\hline
\end{tabular}

*Os números entre parênteses referem-se a proporção entre as gorduras (gordura do leite:óleo de milho).

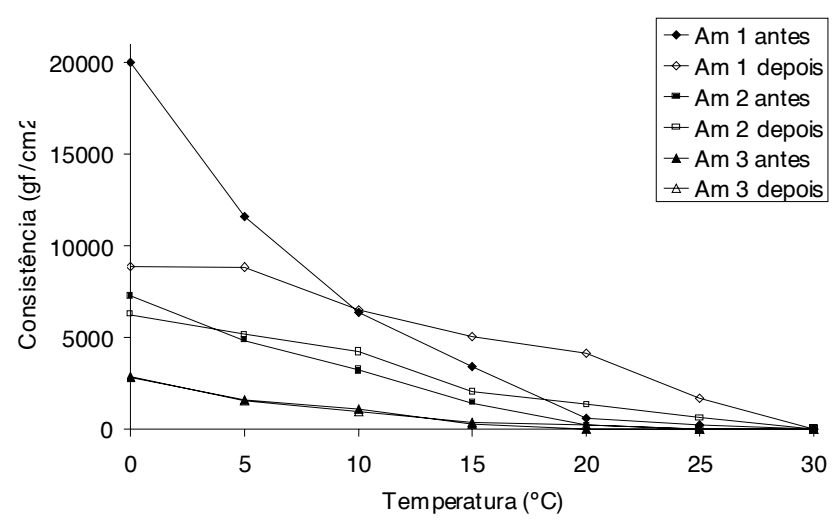

FIGURA 1. Consistência das amostras antes e depois da interesterificação, em função da temperatura.

Os resultados do conteúdo de gordura sólida (CGS) das amostras antes e depois da interesterificação estão contidos na Tabela 5 e representados na Figura 2 para as amostras 1, 2 e 3. O aumento de temperatura provocou a diminuição do CGS de todas as amostras antes e depois da interesterificação, devido à fusão dos cristais. Pode-se observar que a adição de óleo de milho provocou diminuição no CGS, por ser um óleo líquido, e provocou certa linearização do perfil de fusão da gordura do leite, antes e depois do rearranjo. Nota-se que houve uma queda mais intensa do CGS entre 10 e $20^{\circ} \mathrm{C}$ para as amostras 1, 2 e 3, devido à grande proporção de triacilgliceróis que se fundem e se solubilizam nesta faixa [31]. Acima de $20^{\circ} \mathrm{C}$, as inclinações das curvas são menos pronunciadas. Para as amostras 1 e 2, a interesterificação provocou a inversão do comportamento de fusão, entre 10 e $20^{\circ} \mathrm{C}$. 
TABELA 4. Coeficientes de regressão múltipla para a consistência das amostras, antes e depois da interesterificação.

\begin{tabular}{lcccc}
\hline \multicolumn{4}{c}{ Coeficientes } \\
Temperatura ( $\left.{ }^{\mathrm{C}} \mathrm{C}\right)$ & $\beta_{1}$ & $\beta_{2}$ & $\beta_{12}$ & $\mathrm{R}^{2}$ \\
\hline Antes & & & & \\
0 & 18654,2 & 0 & $-34955,0$ & 0,977 \\
5 & 8831,5 & 0 & $-15603,4$ & 0,992 \\
10 & 6241,1 & 0 & $-10450,0$ & 0,994 \\
15 & 3270,9 & 0 & $-6457,6$ & 0,977 \\
20 & 582,2 & 0 & $-1239,9$ & 0,957 \\
Depois & & & & \\
0 & 9210,2 & 0 & $-10874,2$ & 0,994 \\
5 & 7575,3 & 0 & $-12681,9$ & 0,995 \\
10 & 6655,2 & 0 & $-10358,4$ & 0,988 \\
15 & 4801,6 & 0 & $-9672,8$ & 0,983 \\
20 & 3874,0 & 0 & $-8260,4$ & 0,970
\end{tabular}

A Tabela 6 apresenta os coeficientes obtidos por regressão múltipla para o conteúdo de gordura sólida das amostras, antes e depois da interesterificação. O CGS não foi dependente do óleo de milho, mas da gordura do leite e da interação entre eles Os coeficientes $\beta_{12}$ referentes à interação entre os componentes foram negativos, confirmando a interação eutética entre a gordura do leite e o óleo de milho, já observada com as análises de consistência, exceto a $10^{\circ} \mathrm{C}$ depois da interesterificação, quando não foi significativo. O rearranjo provocou diminuição no CGS da gordura do leite (amostra 1) abaixo de $15^{\circ} \mathrm{C}$, mas provocou aumento de 15 a $20^{\circ} \mathrm{C}$, até a fusão total. O mesmo comportamento foi observado por [32] para misturas de gordura do leite e óleo de canola, por interesterificação química.

A interesterificação provocou certa linearização das curvas de CGS em função da temperatura, como mostra a Figura 2. As quedas no CGS decorrentes da interesterificação são devidas à reposição de ácidos graxos saturados por insaturados nos triacilgliceróis da gordura do leite, que contém muitos di- e trissaturados. Devido à presença de duplas ligações, os ácidos graxos insaturados apresentam menor ponto de fusão [31].

TABELA 5. Conteúdo de gordura sólida (\%) das amostras antes e depois da interesterificação, em função da temperatura.

\begin{tabular}{|c|c|c|c|c|c|c|c|c|c|c|c|c|}
\hline $\begin{array}{c}\text { Temperatura } \\
\left({ }^{\circ} \mathrm{C}\right)\end{array}$ & $\begin{array}{r}\text { Amos } \\
(100\end{array}$ & & $\begin{array}{l}\text { Amos } \\
(80: 2\end{array}$ & & $\begin{array}{r}\text { Amos } \\
(60: 4\end{array}$ & & & & $\begin{array}{r}\text { Amo } \\
(20\end{array}$ & & & \\
\hline & Antes & Depois & Antes & Depois & Antes & Depois & Antes & Depois & Antes & Depois & Antes & Depois \\
\hline 10 & 50,6 & 46,3 & 38,1 & 37,1 & 25,5 & 25,6 & 13,8 & 14,0 & 4,1 & 4,6 & 0 & 0 \\
\hline 15 & 35,9 & 34,8 & 24,3 & 25,5 & 14,2 & 14,7 & 6,7 & 6,3 & 1,5 & 0,2 & - & - \\
\hline 20 & 17,8 & 22,9 & 12,0 & 15,6 & 7,5 & 7,6 & 3,7 & 3,1 & 0,3 & 0,2 & . & - \\
\hline 25 & 12,0 & 17,2 & 8,0 & 11,0 & 4,5 & 4,9 & 1,5 & 0 & 0,1 & 0 & - & - \\
\hline 30 & 4,1 & 9,7 & 2,5 & 5,0 & 0,9 & 1,5 & 0 & - & 0 & - & - & - \\
\hline 35 & 0,3 & 3,7 & 0 & 1,2 & 0 & 0 & - & - & - & - & - & - \\
\hline 40 & 0 & 0 & . & 0 & - & . & . & . & . & . & . & - \\
\hline
\end{tabular}

*Os números entre parênteses referem-se a proporção entre as gorduras (gordura do leite:öleo de milho).
TABELA 6. Coeficientes de regressão múltipla para o conteúdo de gordura sólida das amostras, antes e depois da interesterificação

\begin{tabular}{lcccc}
\hline & \multicolumn{3}{c}{ Coeficientes } & \\
\cline { 2 - 3 } Temperatura $\left({ }^{\circ} \mathrm{C}\right)$ & $\beta_{1}$ & $\beta_{2}$ & $\beta^{2}$ \\
\hline Antes & & & & \\
10 & 51,4 & 0 & $-23,9$ & 0,999 \\
15 & 36,2 & 0 & $-31,3$ & 0,999 \\
20 & 17,9 & 0 & $-14,2$ & 0,999 \\
25 & 12,2 & 0 & $-12,5$ & 0,999 \\
30 & 4,2 & 0 & $-6,4$ & 0,996 \\
Depois & & & & \\
10 & 47,5 & 0 & 0 & 0,998 \\
15 & 35,9 & 0 & $-28,7$ & 0,996 \\
20 & 23,4 & 0 & $-25,0$ & 0,998 \\
25 & 17,7 & 0 & $-24,7$ & 0,995 \\
30 & 9,6 & 0 & $-16,7$ & 0,996 \\
35 & 3,4 & 0 & $-7,7$ & 0,961 \\
\hline
\end{tabular}

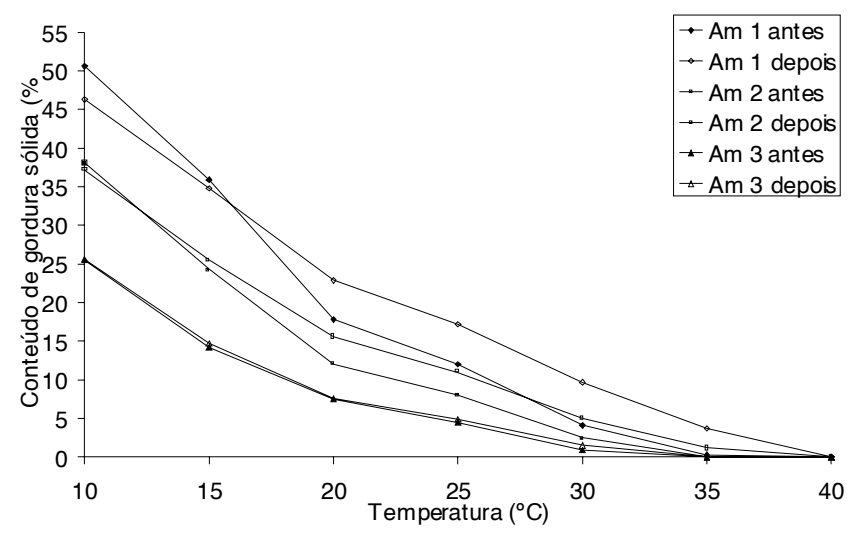

FIGURA 2. Conteúdo de gordura sólida das amostras antes e depois da interesterificação, em função da temperatura.

O comportamento reológico da gordura do leite, ou seja, todos os fenômenos relacionados a escoamento e deformação, são influenciados por um grande número de variáveis. Normalmente, muitas delas operam ao mesmo tempo e torna-se dificil separar estes fatores, de modo que seu efeito relativo possa ser estudado. Entre estes fatores estão temperatura, conteúdo de gordura sólida, tamanho, distribuição do tamanho e forma dos cristais, forças de interação entre as partículas e tratamento mecânico dado à gordura. Destes, um dos fatores mais importantes que afetam a consistência é o teor de sólidos da gordura do leite [9]. Assim, foi efetuada uma correlação entre os valores experimentais de conteúdo de gordura sólida e consistência das amostras às temperaturas de 10 a $30^{\circ} \mathrm{C}$ por regressão linear simples. Os coeficientes da regressão e os valores de $\mathrm{R}^{2}$ estão apresentados na Tabela 7. A equação da reta correspondente é a seguinte:

$$
\mathrm{y}=\mathrm{ax}+\mathrm{b}
$$


onde: $y=$ conteúdo de gordura sólida (\%)

$x=$ consistência $\left(\mathrm{gf} / \mathrm{cm}^{2}\right)$

$\mathrm{a}=$ coeficiente angular

$\mathrm{b}=$ coeficiente linear

Os resultados mostraram que houve relação linear significativa a $\mathrm{p}<0,05$ entre as propriedades de consistência e conteúdo de gordura sólida para as amostras 1 , 2 e 3, tanto antes quanto após a interesterificação. As amostras 4, 5 e 6 não apresentaram valores de consistência suficientes para se estabelecer uma correlação. $\mathrm{O}$ coeficiente linear representa o conteúdo de gordura sólida quando a consistência é igual a zero. Os valores vão de 9,7 a 5,2\% antes da interesterificação e de 7,6 a 3,3\% para as correspondentes amostras interesterificadas. Isto mostra que, quando o teor de sólidos na gordura é muito baixo, o material apresenta-se como um liquido viscoso, ao invés de se comportar como uma gordura plástica. Quando a proporção de gordura no estado sólido é maior que cerca de $10 \%$, o óleo liquido é imobilizado pela matriz cristalina, tornando a gordura plástica [25].

TABELA 7. Coeficientes de regressão linear simples para conteúdo de gordura sólida e consistência antes e depois da interesterificação.

\begin{tabular}{lccc}
\hline \multirow{2}{*}{ Amostra } & \multicolumn{3}{c}{ Coeficientes } \\
\cline { 2 - 3 } & $\mathrm{a}$ & $\mathrm{B}$ & $\mathrm{R}^{2}$ \\
\hline Antes & 0,01 & 9,69 & 0,957 \\
1 & 0,01 & 7,08 & 0,955 \\
2 & 0,02 & 5,16 & 0,902 \\
3 & & & \\
Depois & 0,01 & 7,63 & 0,917 \\
1 & 0,01 & 6,12 & 0,970 \\
2 & 0,02 & 3,33 & 0,962 \\
3 & & & \\
\hline
\end{tabular}

TABELA 8. Conteúdo de gordura sólida da mistura 70:30, antes e depois da interesterificação, em função da temperatura.

\begin{tabular}{ccc}
\hline & \multicolumn{2}{c}{ Conteúdo de gordura sólida (\%) } \\
\hline Temperatura $\left({ }^{\circ} \mathrm{C}\right)$ & Antes & Depois \\
10 & 31,0 & 33,3 \\
15 & 18,8 & 19,1 \\
20 & 9,5 & 11,1 \\
25 & 5,9 & 7,2 \\
30 & 1,6 & 3,2 \\
35 & - & 0,8 \\
\hline
\end{tabular}

O conteúdo de gordura sólida da mistura de proporção 70:30 de gordura do leite:óleo de milho, antes e depois da interesterificação, foi calculado a partir dos coe- ficientes da regressão múltipla e está representado na Tabela 8. Os cálculos confirmam seu comportamento intermediário às amostras 2 e 3, o que pode ser observado comparando-se estes valores com os da Tabela 5. Esta proporção, tanto antes quanto depois da reação, resultaria em produto com teor de sólidos ideal à temperatura de refrigeração (cerca de $30 \%$ ), cuja plasticidade resistiria até aproximadamente $20^{\circ} \mathrm{C}$. A mistura interesterificada apresentou maior CGS em todas as temperaturas, mostrando-se um pouco mais consistente a temperaturas mais altas. Este comportamento é desejado em um pais de clima quente, como o Brasil [14].

\section{4 - CONCLUSÕES}

Foram obtidos lipídios estruturados contendo teores de até $22 \%$ de ácidos graxos poliinsaturados $\omega-6$ pela adição de até $40 \%$ de óleo de milho à gordura do leite, que originalmente possuía apenas cerca de $2 \%$. As misturas apresentaram plasticidade própria de um spread, propriedade que pôde ser melhorada ainda mais com a reação de interesterificação. A consistência e o conteúdo de gordura sólida mostraram-se dependentes da gordura do leite, antes e após a interesterificação. O óleo de milho, por ser liquido, não colaborou estatisticamente para estas propriedades. Os coeficientes significativos relativos às interações entre os componente foram sempre negativos para ambas as propriedades, demonstrando efeito antagônico, característico de interações eutéticas entre gorduras. Os resultados mostraram relação linear significativa entre o conteúdo de gordura sólida e a consistência das amostras.

\section{5 - REFERÊNCIAS}

[1] AMERICAN OIL CHEMISTS' SOCIETY. Official methods and recommended practices of the AOCS. 4. ed. Champaign, 1990. Methods Ce 1-62 and Cd 16b-93.

[2] BALCÃO, V. M., KEMPPINEM, A., MALCATA, F. X., KALO, P. J. Modification of butterfat by selective hydrolysis and interesterification by lipase: Process and product characterization. Journal of the American Oil Chemists' Society, v. 75, n. 10, p. 1347-1358, 1998.

[3] BALCÃO, V. M., MALCATA, F. X. Interesterification and acidolysis of butterfat with oleic acid by Mucor javanicus lipase: Changes in the pool of fatty acid residues. Enzyme and Microbial Technology, v. 22, n. 6, p. 511-519, 1998.

[4] BRASIL, Leis, decretos, etc. Portaria do Ministério da Agricultura, Abastecimento e Reforma Agrária (MAARA) $n^{\circ} 146$ de 07 de março de 1996. Aprova padrões microbiológicos, físicos e químicos para leite e derivados. Diário Oficial. Brasília, 11 de março de 1996, seção 1, p. 3977-3986.

[5] BRASIL, Leis, decretos, etc. Resolução n. 482 de 23 de setembro de 1999. Disponivel em: http:// www.anvisa.gov.br/legis/resol/482_99.htm. Acesso em: 30 de outubro de 2001.

[6] CHRYSAM, M. M. - Table spreads and shortenings, L. In: APPLEWHITE, T. H. (Ed.) Bailey's industrial oil and fat products. 4a. ed. New York: Wiley-Interscience, 1985. v. 3, p. 41-126.

[7] D’Agostini, D., FERRAZ, R.C., GIOIELLI, L.A. Consistência de misturas binárias e ternárias de gorduras de pal- 
ma, palmiste e triacilgliceróis de cadeia média. Revista Brasileira de Ciências Farmacêuticas, v.36, n. 1, p. 147155, 2000

[8] deMAN, J. M. Consistency of fats: a review. Journal of the American Oil Chemists' Society, v. 60, n. 1, p. 82-87, 1983.

[9] deMAN, J. M. Physical properties of milk fat. Journal of Dairy Science, v. 47, p. 1194-1200, 1964.

[10] DUNCAN, S. E. Dairy products: The next generation. Altering the image of dairy products through technology. Journal of Dairy Science, v. 81, n. 4, p. 877-883, 1998.

[11] FITZPATRICK, K.C. Stanol ester products face U. S. hurdles. INFORM, v. 10, n. 2, p. 172-177, 1999

[12] GARCIA, D. J. Omega-3 long-chain PUFA nutraceuticals. Food Technology, v. 52, n. 6, p. 44-49, 1998.

[13] GIOIELLI, L. A. Interesterificação de óleos e gorduras. Revista Engenharia de Alimentos, v. 4, n. 21, p. 22-24, 1998.

[14] GIOIELLI, L.A. Misturas binárias e ternárias de gorduras hidrogenadas na formulação de margarinas. São Paulo, 1996. 253 p. Tese de Livre-Docência - Faculdade de Ciências Farmacêuticas, Universidade de São Paulo (USP).

[15] GUNSTONE, F. D., HARWOOD, J. L., PADLEY, F. B. 1986 The lipid handbook. Chapman and Hall, London.

[16] HAIGHTON, A. J. The measurement of the hardness of margarine and fats with cone penetrometers. Journal of the American Oil Chemists' Society, v. 36, n. 8, p. 345-348, 1959.

[17] HARTMAN, L., LAGO, R. C. A. Rapid preparation of fatty acid methyl esters from lipids. Laboratory Practice, v. 22, p. 475-476, 494, 1973.

[18] HASLER, C.M. Functional foods: Their role in disease prevention and health promotion. Food Technology, v. 52, n. 11, p. 63-70, 1998.

[19] HAUMANN, B. F. Health benefits may be key for foods in the future. INFORM, v. 10, n. 1, p. 18, 1999.

[20] HUI, Y. H. (Ed.) Bailey's industrial oil and fat products. New York, 1996, v. 2, p. 125-158.

[21] JENSEN, R. G., FERRIS, A. M., LAMMI-KEEFE, C. J. Milk fat composition, function, and potential for change: The composition of milk fat. Journal of Dairy Science, v. 74, p. 3228-3243, 1991.

[22] JIMÉNEZ-FLORES, R. Trends in research for alternate uses of milk fat. Journal of Dairy Science, v. 80, n. 10, p. 2644 - 2650, 1997.

[23] KAWANARI, M. Butter's characteristics: Effect of processing. INFORM, v. 7, n. 10, p. 1104-1110, 1996.

[24] LAJOLO, F. Alimentos funcionais: Legislação brasileira. In: I Seminário Internacional sobre Alimentos Funcionais, São Paulo, SP, 1999. CD-ROM.

[25] LARSSON, K. A. 1994. Lipids: molecular organization, physical functions and technical applications. The Oily Press, Dundee.
[26] LIEVENSE, L. C. Plant sterols: A new way to effectively reduce cholesterol. In: I Seminário Internacional sobre Alimentos Funcionais, São Paulo, SP, 1999. CDROM.

[27] MARANGONI, A. G., ROUSseaU, D. The influence of chemical interesterification on physicochemical properties of complex fat systems. 1. Melting and crystalization. Journal of the American Oil Chemists' Society, v. 75, n. 10, p. 1265-1271, 1998a.

[28] MARANGONI, A. G., ROUSSEAU, D. The influence of chemical interesterification on physicochemical properties of complex fat systems. 1 Melting and crystallization. Journal of the American Oil Chemists' Society, v. 75, n. 10, p. 1265-1271, 1998b.

[29] MOLKENTIN, J., PRECHT, D. Validation of a gaschromatographic method for the determination of milk fat contents in mixed fats by butyric acid analysis. European Journal of Lipid Science and Technology, v. 102, n. 3, p. 194-201, 2000.

[30] ROSSELL, J. B. Interactions of triglycerides and of fats containing them. Chemistry and Industry, n. 9, p. 832835, 1973.

[31] ROUSSEAU, D., FORESTIERE, K., HILL, A. R., MARANGONI, A. G. Restructuring butterfat through blending and chemical interesterification. 1. Melting behavior and triacylglycerol modifications. Journal of the American Oil Chemists' Society, v. 73, n. 8, p. 963-972, 1996a.

[32] Rousseau, D., Hill, A. R., MARAngoni, A. G. Restructuring butterfat through blending and chemical interesterification. 3. Rheology. Journal of the American Oil Chemists' Society, v. 73 , n. 8, p. 983989, 1996b.

[33] ROZENAAL, A. Interesterification of oils and fats. INFORM, v. 3, n. 11, p. 1232-1237, 1992.

[34] SIMÕES, I. S., GIOIELLI, L. A. Cristalização e polimorfismo de gorduras. Óleos \& Grãos, v. 8, n. 50, p. 20-29, 1999.

[35] SREENIVASAN, B. Interesterification of fats. Journal of the American Oil Chemists' Society, v. 55, n. 11, p. 796-805, 1978.

[36] TIMMS, R. E. Phase behaviour of fats and their mixtures. Progress in Lipid Research, v. 23, n. 1, p. 1-38, 1984.

[37] WIEDERMANN, L. H. Margarine and margarine oil, formulation and control. Journal of the American Oil Chemists' Society, v. 55, n. 11, p. 823-829, 1978.

\section{6 - AGRADECIMENTOS}

À Fundação de Amparo à Pesquisa do Estado de São Paulo - FAPESP e ao Conselho Nacional de Desenvolvimento Científico e Tecnológico - CNPq, pelo auxílio financeiro e pelas bolsas concedidas aos autores. 